\title{
A Cleverly Designed Novel Lipid Nanosystem: Targeted Retention, Controlled Visual Drug Release, and Cascade Amplification Therapy for Mammary Carcinoma in vitro [Corrigendum]
}

Zhao XZ, Zhang $\mathrm{W}$, Cao $\mathrm{Y}$, et al. Int $J$ Nanomedicine. 2020;15:3953-3964.

The authors have advised due to an error that occurred inadvertently at the time of figure assembly, Figure $2 \mathrm{M}$ on page 3956 is incorrect.

The correct Figure 2 is shown below.
A

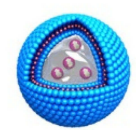

B

LIPO:LIP@AQ4N@HMME@PFH

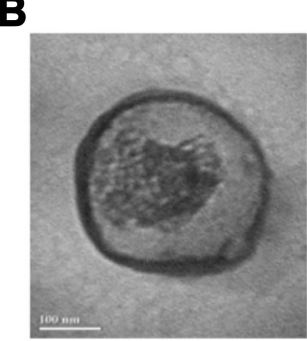

$\mathbf{F}$

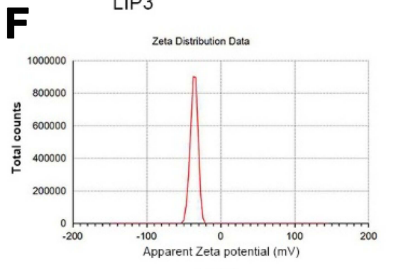

G

童.

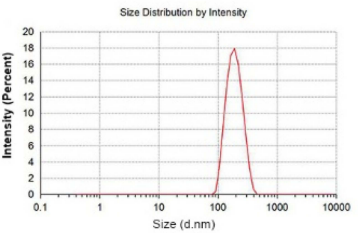

H

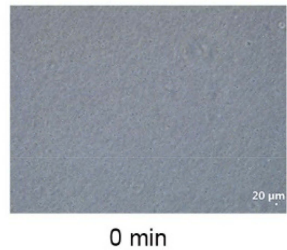

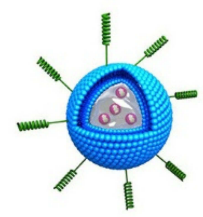

C

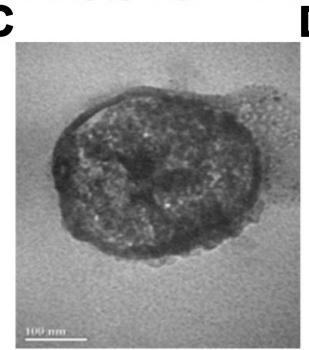

LIP3 phase contrast

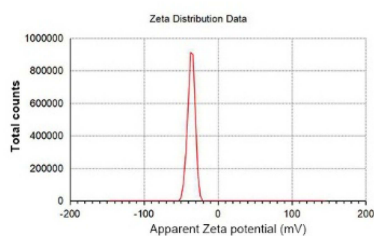

LIP2

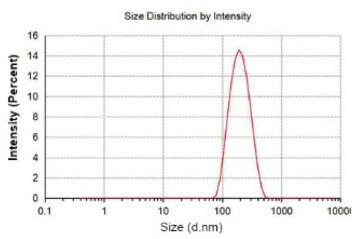

LIP2

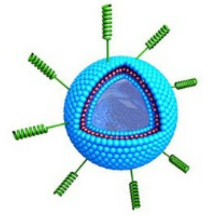

D

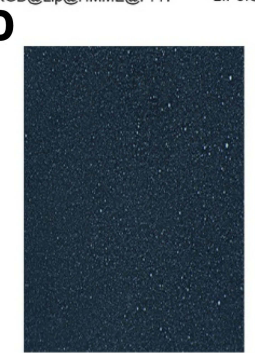

LIP3

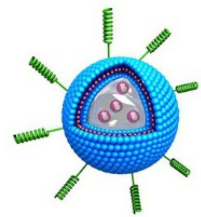

IP3:C-RGD@LIP@AQ4N@HMME@PFH $\mathbf{E}$

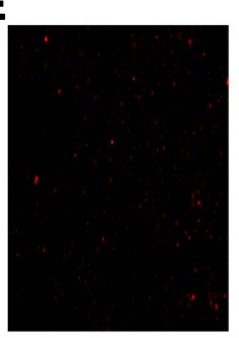

LIP3

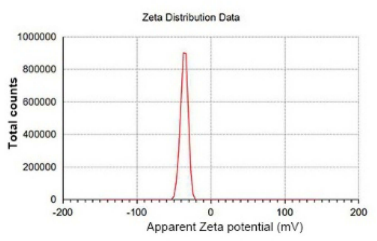

LIP3

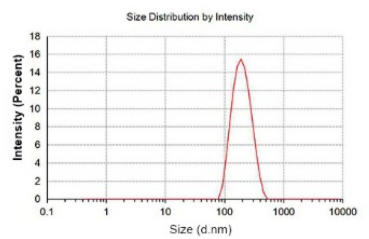

LIP3 
I

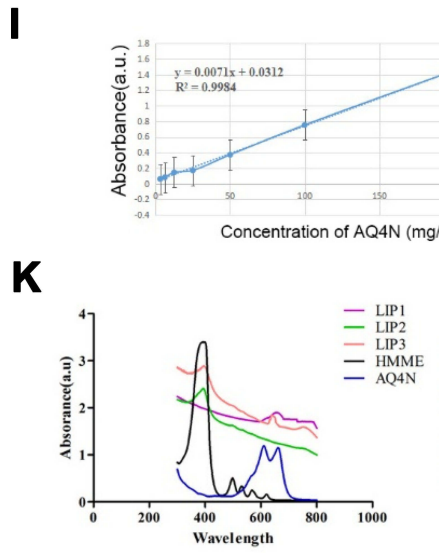

$\mathbf{J}$

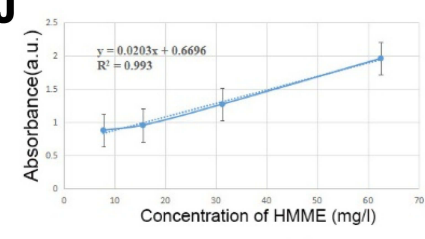

M
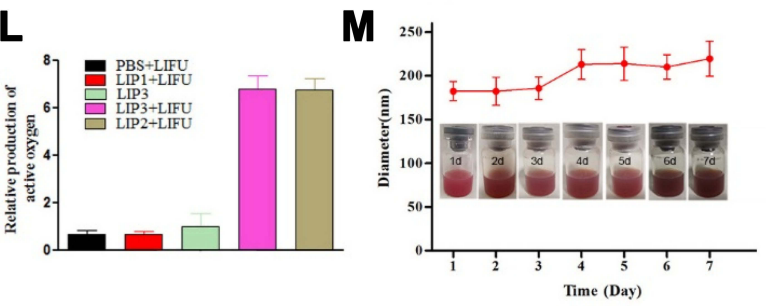

Figure 2 General physicochemical properties of LIP3. (A) Structure illustration of LIP0, LIPI, LIP2, and LIP3. (B) TEM of LIP3. (C) Phasing sign TEM of LIP3. (D) Light microscopy image of LIP3. (E) Fluorescence microscope image of Dil-stained LIP3 NPs ( $\times 400)$. (F) Zeta potential of LIPI, LIP2, and LIP3. (G) Size of LIPI, LIP2, and LIP3. (H) LIP3 light microscopy image of the sonogenic phase transition ( $\times$ 400). (I) Correlation between concentration of AQ4N and absorbance. (J) Correlation between concentration of HMME and absorbance. (K) UV-vis-NIR absorbance spectra of LIPI, LIP2, and LIP3 and free HMME, AQ4N. (L) Relative production of active oxygen of LIP3. (M) LIP3 size distribution with prolonged time duration. Insert: digital photos of the LIP3 NPs dispersed in PBS (2.5 mg/mL).

A
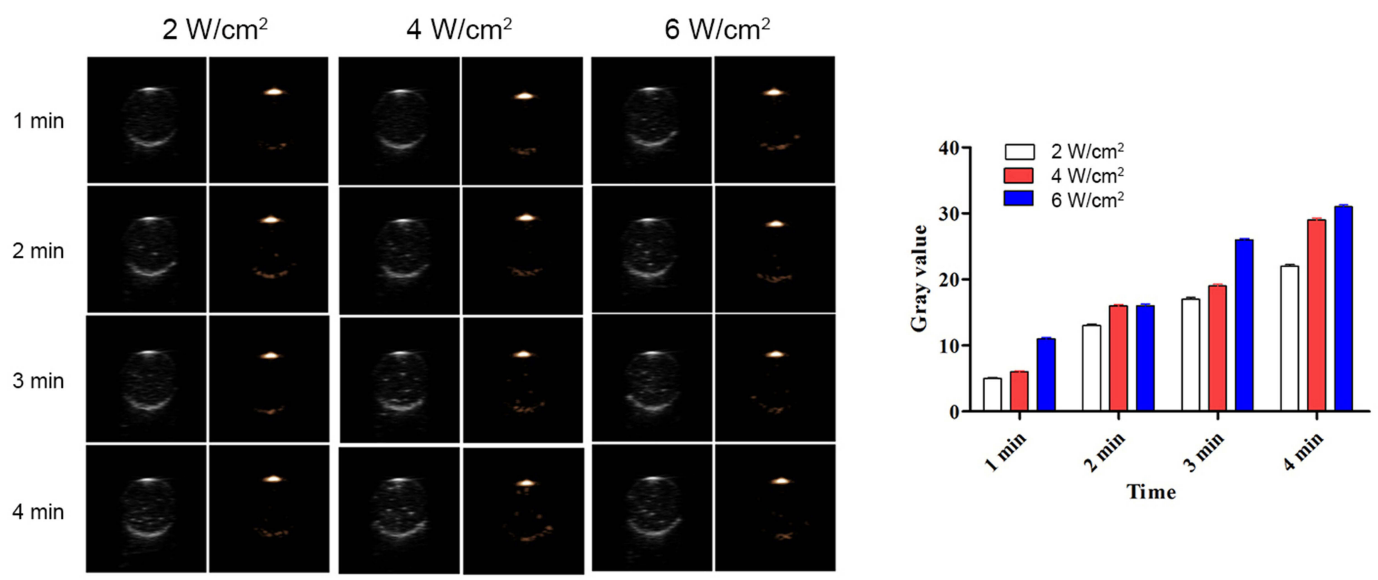

B
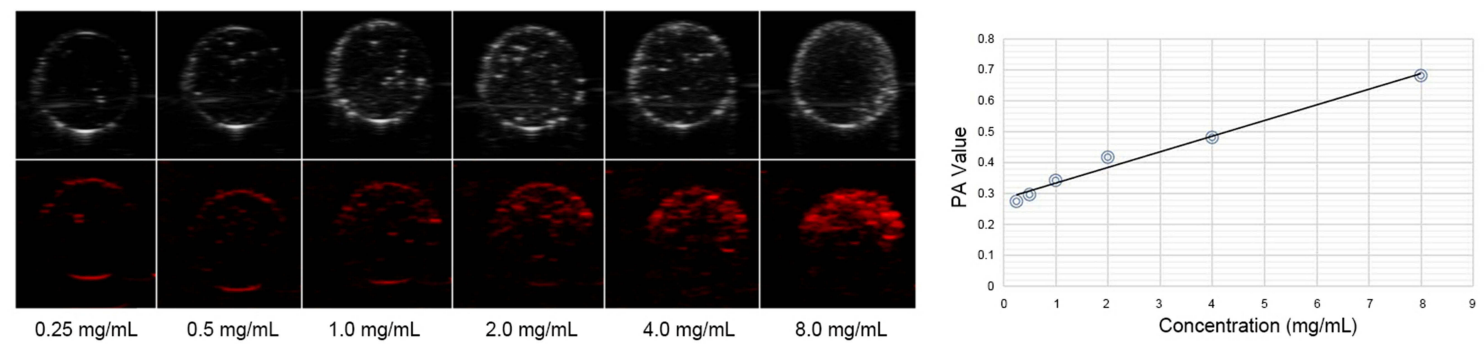

Figure 3 LIP3 bimodal imagining in vitro. (A) LIP3 CEUS imaging and grey value histogram of different groups in vitro. (B) LIP3 PA imaging and PA value scatter diagram with different concentrations in vitro. 


\section{Publish your work in this journal}

The International Journal of Nanomedicine is an international, peerreviewed journal focusing on the application of nanotechnology in diagnostics, therapeutics, and drug delivery systems throughout the biomedical field. This journal is indexed on PubMed Central, MedLine, CAS, SciSearch ${ }^{\mathbb{R}}$, Current Contents ${ }^{\mathbb{B}} /$ Clinical Medicine,
Journal Citation Reports/Science Edition, EMBase, Scopus and the Elsevier Bibliographic databases. The manuscript management system is completely online and includes a very quick and fair peer-review system, which is all easy to use. Visit http://www.dovepress.com/ testimonials.php to read real quotes from published authors. 